XVIII. Aus der inneren Abtheilung des städtischen Krankenhauses am Urban zu Berlin. ${ }^{1}$ )

\section{Beobachtungen über die Anwendung des Koch'schen Heilverfahrens.}

\section{Von A. Fraenkel.}

Meine Herren! Wenn ich heute, der an mich ergangenen Aufforderung des Vorsitzenden dieser Gesellschaft Folge leistend, das Wort ergreife, um Ihnen meine bisherigen Beobachtungen und Erfahrungen über die Anwendung des Koch'schen Mittels bei den tuberculösen Patienten der inneren Station des städtischen Krankenhauses am Urban mitzutheilen, so befinde ich mich in einer ähnlichen Lage wie der Vorredner, Herr College Lindner und die meisten derjenigen Herren Collegen, welche in den letzten beiden Wochen über den gleichen Gegenstand in den verschiedenen wissenschaftlichen Gesellschaften und Vereinen unserer Stadt gesprochen haben. Es sind erst 16 Tage verflossen, seitdem wir in den Besitz des Impfstoffes gelangt sind und Injectionen mit demselben vornehmen; Sie werden daher von vornherein darauf verzichten müssen, dass ich Thnen bereits über erzielte Heilerfolge bei den mit Tuberculose innerer Organe behafteten Kranken Mittheilung mache. Dazu ist der verstrichene Zeitraum viel zu gering, und es werden allem Anschein nach eine ganze Reihe von Monaten darüber vergehen, bis wir in der Lage sein werden, uns ein eigenes Urtheil darüber $z u$ bilden, was das Ko ch'sche Mittel speciell in der Bekämpfung der tuberculösen Lungenphthise, welche naturgemäss das Hauptcontingent meiner Kranken ausmacht, zu leisten vermag. Nichtsdestoweniger habe ich dem an mich herangetretenen Ersuchen ohne Bedenken Folge leisten zu müssen geglaubt, erstens, weil für alle diejenigen, die noch nicht in der glücklichen Lage sich befinden, die Injectionsflüssigkeit zu besitzen, es von bohem Werthe sein muss, Erfahrungen gerade über die erste Anwendung und Wirkungsweise des mit so merkwürdigen Eigenschaften ausgestatteten Mittels kennen zu lernen; zweitens, weil speciell das meiner Beobachtung zu Gebote stehende Krankenmaterial kein einförmiges, sondern in Bezug auf die Natur der Affectionen ein überaus mannichfaches und instructives ist.

Bis zum Ende voriger Woche waren 32 Patienten von uns in Behandlung genommen worden. Davon sind 27 mit unzweifelhafter Lungenphthise behaftet; von den restirenden 5 leidet einer an einer anscheinend abgelaufenen (vorher operativ behandelten) tuberculösen Peritonitis, einer an beiderseitiger lymphomatőser Hyperplasie der Cervicaldrüsen, zwei an Pleuritis, nnd einer an Bronchiektasenbildung in der rechten Lunge mit Verdichtung des zwischen den erweiterten Luftröhrenästen belegenen Parenchyms. Der letztere Fall, welcher eine 50jährige Frau betrifft, bei der sich das Leiden vielleicht auf der Basis einer voraufgegangenen Rippenfellentzündung entwickelt hat, diente uns gewissermaassen als Controllfall. $\mathrm{Zu}$ keiner Zeit wurden in dem Auswurfe der Kranken Bacillen oder elastische Fasern gefunden, und dementsprechend haben wir bei derselben auch nicht die Erscheinungen einer deutlich ausgesprochenen "Reaction" wahrgenommen, so dass dieser Fall von vornherein aus der weiteren Betrachtung ausscheiden kann. Von den an tuberculöser Lungenphthise leidenden 27 Patienten bietet einer zugleich die Erscheinungen einer Otitis media perforativa tuberculosa, drei diejenigen einer (vermuthlich tuberculősen) Pleuritis dar; bei einem vierten Kranken hat sich das Lungenleiden im Gefolge von Diabetes entwickelt, und endlich leiden drei weitere Patienten zugleich an Laryngophthisis. Mit Ausnahme eines 12 jährigen Mädchens haben die Kranken, unter welchen sich 24 mănnliche und 8 weibliche befinden, sämmtlich das 16. Lebensjahr überschritten. Ich will hier gleich voranschicken, dass ich bei der Auswahl der Lungenkranken so vorgegangen bin, dass ich mit Ausnahme von dreien, welche ich zu den „mittelschweren" zăhle, nur leichte Fälle genommen habe,

1) Nach einem Vortrag, gehalten in der Gesellschaft für öffentliche $\mathrm{G}_{\theta}$ sundheitspflege am 8. December 1890. d. h. solche, bei welchen vor allem durch die physikalische Untersuchung keine deutlichen Cavernensymptome nachzuweisen sind, die Veränderungen sich überhaupt nur auf Dämpfungen sehr mässiger Intensität, meist auf der einen Seite resp. Spitze, beschränken, der Ernährungszustand noch ein relativ günstiger, mit einem Wort die Krankheit noch nicht weit vorgeschritten ist, sondern sich vielmehr in ihrem Anfange befindet. Ich lege ein besonderes Gewicht darauf, das hervorzuheben, da. nur an solchen Fällen ein klarer Ueberblick darüber zu gewinnen ist, was das Mittel bezüglich der definitiven Heilbarkeit der Lungenphthise zu leisten im Stande ist. Auf die Entscheidung dieser Frage aber kommt es für mich als internen Mediciner in erster Linie an, und ich brauche mit Rücksicht auf die zweite Publication Koch's nicht des ausführlicheren darzulegen, weshalb die in vorgeschritteneren Stadien des Leidens befindlichen Kranken im grossen und ganzen ungeeignet sind, um zur Lösung derselben herangezogen zu werden, ganz abgesehen davon, dass bei ihnen selbst eine vorsichtige Behandlung nicht immer sich ganz ungefährlich gestalten dürfte. Gleichfalls im Interesse der Exaktheit und Uebersicht der Beobachtungsreihe habe ich es vorgezogen, zunächst nur eine beschränkte Zahl von Patienten in Behandlung zu nehmen, wobei mir durch das reichliche Material der inneren Abtheilung des städtischen Krankenhauses am Urban. die passende Auswahl noch besonders erleichtert wurde. Von jedem Kranken ist vor Beginn der Einspritzungen das Sputum auf das genaueste untersucht worden; es wird zu Anfang mindestens einen Tag um den anderen, in vielen Fällen sogar tagtäglich von dem Auswurf des betreffenden Patienten ein Doppelpräparat gemacht und dasselbe aufbewahrt, damit wir jederzeit im Besitze der Vergleichsobjecte uns befinden. Die Färbung des Sputums geschieht mit der so sicheren und expediten Gabbet'schen Methode; doch will ich gleich hier bemerken, dass der nicht gelungene Nachweis von Bacillen mit derselben noch keineswegs deren Fehlen im Sputum beweist. Will man ganz sicher gehen, so muss in solchen Fällen mit negativem Ausfall der Untersuchung noch die Sedimentirungs- oder Biedert'sche Untersuchungsmethode zu Hilfe gezogen werden, welche uns gewissermaassen gestattet, die Bacillen eines grősseren Quantums von Auswurf im Betrage von 1-2 Esslöffeln auf $1 / 2-1 \mathrm{ccm}$ Flüssigkeit zu concentriren, und welche daher an Sicherheit die übrigen Methoden weitaus übertrifft. Noch allemal, wenn uns bisher die anderen Untersuchungsmethoden, die Gabbet'sche, Ehrlich'sche u. s. w. im Stiche liessen, konnten wir durch das Biedert'sche Verfahren unschwer Bacillen nachweisen. Es ist das ein Controllverfahren, auf dessen zuverlăssige und entscheidende Bedeutung ich die Herren Collegen nicht nachdrücklich genug verweisen kann, da - abgesehen von der viel umständlicheren Prüfung durch die Thierimpfung - nur so sich Täuschungen vermeiden lassen. Dass bei jedem Kranken fortlaufend genaue Notizen über die Menge des Auswurfs, die physikalischen Symptome, das Verhalten der Athmung, des Harns, Körpergewichtes u. s. w. gemacht werden, versteht sich von selbst. All das erfordert aber viel Mühe und Zeit, und in Folge dessen kann auch die Zahl der behandelten Kranken zunächst nur eine beschränkte sein.

Bevor ich zur Besprechung der von den Kranken nach erfolgter Injection dargebotenen Erscheinungen übergehe, habe ich noch einiges über die Dosirung des Mittels vorauszuschicken. Als wir am Vormittage des 22. November in Gegenwart einer grösseren Zahl von Collegen sechs unserer mit Lungenphthise behafteten Kranken die erste Injection von 0,001 g Substanz machten, waren wir nicht wenig überrascht, am Abende bei keinem derselben eine erheblichere Reaction wahrzunehmen; bei fünf hatte sich die Temperatur gar nicht gehoben, bei einem, welcher schon vorher gefiebert hatte, war nur eine mässige Erhöhung anf $38,5^{\circ} \mathrm{C}$ erfolgt. Auch die Verdoppelung der anfänglichen Dosen hatte nur einen mässigen Erfolg. Infolge dessen gingen wir in den nächsten Tagen etwas dreister vor. d. h. wir stiegen alsbald von 2 resp. 3 anf 5 Milligramm. Jedoch sind wir von diesem beschleunigten Ver- 
fahren alsbald zu der von Koch selbst mit grosser Präcision und Klarheit gegebenen Vorschrift des vorsichtigen Steigens zurückgekehrt, nachdem sich bei einem unserer mittelschweren Fälle eine ziemlich lebhafte, mehrere Tage andauernde Dyspnoe entwickelt hatte, bei einem anderen Kranken, welcher allerdings schon früher mehrfach Blut ausgeworfen hatte, anscheinend im Anschluss an die Einspritzungen eine neue Haemoptoë erfolgt war. Wir gehen jetzt ausschliesslich so vor, dass wir mit einem Milligramm beginnend zunächst nur milligrammweise steigen und erst dann von der einmal erreichten Dosis zu der folgenden übergehen, wenn deren Wirkung hinsichtlich des temperatursteigernden Effectes vollständig abgeklungen, bezw. die Körperwärme auf den zuvor beobachteten Stand zurückgegangen ist. Das Fortschreiten zu höheren Gaben gestaltet sich dabei, wie Sie am besten aus den Ihnen nachher vorzulegenden Temperaturcurven ersehen werden, je nach der Individualität des Falles mit verschiedener Geschwindigkeit. Wir haben Patienten, bei denen wir noch vor Ablauf des achten Tages bis zu 0,01 gelangt sind, und zwar ohne irgend einen allarmirenden Zwischenfall, während in anderen Fällen die gleiche Dósenhöhe noch nicht nach 14 Tagen erreicht wurde. In manchen Fällen empfahl es sich sogar, von einer bereits erreichten höheren Gabe, etwa von $0,006-0,007$, auf eine niedrigere, beispielsweise 0,003 , für kurze Zeit zurückzugehen, weil sich die Patienten zu matt und angegriffen $z u$ fühlen begannen. Es ist hierbei von Wichtigkeit zu wissen, dass man sich mit Vortheil ausser der temperatursteigernden Wirkung des Mittels noch anderer Indikatoren für die Bemessung der Dosirung bedienen kann. Dazu rechne ich vor allem die Pulsfrequenz. Patienten, welche eine nur mässige Steigerung derselben nach wiederholter Application darboten, und bei denen die Frequenz nie 120 nach den Injectionen überschritt, sondern im Gegentheil sich mehr oder weniger weit unterhalb dieser Grenzzahl, zwischen 80 bis 100 bewegte, gewahrten im allgemeinen die Voraussage, dass sie relativ grosse Dosen vertragen würden; bei ihnen kann man daher auch bald etwas dreister vorgehen, während eine von vornherein sehr bedeutende Frequenzzunahme des Pulses bis auf 130 Schläge und darüber zur Vorsicht mahnen muss. Daneben kommt viel auf die Athmung und auf die subjective Angabe über die Beschaffenheit derselben an. Insbesondere rathe ich, auf der Hut $z u$ sein, sobald die Zahl der Respirationen über 40 in der Minute steigt, und die Patienten über irgend wie lebhaftere Beklemmung klagen oder gar objectiv die Erscheinungen von Dyspnoe darbieten; in solchen Fallen sollte man stets die Injectionen zunächst aussetzen und abwarten, bis die genannten Erscheinungen verschwnnden sind, um dann zu der vorherigen oder lieber noch einer niedrigeren Dosis zurückzukehren. Ist erst einmal die Dosis von 0,01 erreicht; so thut man überhaupt gut, auch bei völligem Wohlbefinden der Kranken, denselben $a b$ und an Ruhe zu gönnen und für ein oder zwei Tage die Injectionen ganz $\mathrm{zu}$ unterbrechen. Ueber 0,04 sind wir bisher bei unseren Lungenkranken überhaupt noch nicht hinausgegangen, und das höchste bis jetzt injicirte Quantum, welches bei dem Patienten mit abgelaufener Peritonitis tuberculosa zur Anwendung kam, betrug 0,05. Meine Absicht ist ferner, bei den Phthisikern die Dosis von 0,1 , sobald dieselbe erreicht sein wird, nicht zu überschreiten, sondern diese zunächst als Maximaldosis festzuhalten und auch sie nur in Gestalt vereinzelter, durch mehrtägige Ruhetage unterbrochener Injectionen anzuwenden. Das aber will ich mir nicht versagen, an dieser Stelle mit besonderer Schärfe hervorzuheben, dass von ein er ambulanten Behandlung - wenigstens in den ersten Wochen - ganz abstrahirt werden sollte. Die Kranken müssen in dieser ersten Periode mindestens zweimal täglich von dem Arzte gesehen werden; denn wir dürfen nicht vergessen, dass wir es mit einem Mittel zu thun haben, welches toxische Nebenwirkungen besitzt, deren Intensität im einzelnen Falle sich nicht immer mit Sicherheit im voraus bemessen lässț. Nichts könnte der Behandlungsmethode mehr Schaden zufügen, als die Ausserachtlasssung der von ihrem Entdecker selbst in so wohl überlegter Weise an- empfohlenen nothwendigen Vorsichtsmaassregeln. Erst wenn eine complete Gewöhnung der Patienten eingetreten, d. h. wenn die-oben angeführte Maximaldosis erreicht ist, beabsichtige ich die Patienten in ihre Häuslichkeit wieder zu entlassen, sie dann mit zweimal wöchentlich im Krankenhause zu wiederholenden Injectionen weiter zu behandeln und so zugleich das Hospital selbst von einer übermässigen Belegung mit tuberculösen Kranken zu entlasten.

Ich komme nun zu den Allgemeinerscheinungen, welche die von mir behandelten Kranken darboten, und werde mich dabei nur auf die Hervorhebung derjenigen Punkte beschränken, welche im Vergleiche mit den Erfahrungen anderer Aerzte mir besonders bemerkenswerth erscheinen. Während, wie Sie wissen, bei der Tuberculose der Haut und Gelenke in der Mehrzahl der Fälle - auf die auch hier vorkommenden Abweichungen hat Herr College Lindner uns eben erst aufmerksam gemacht - bereits die erste Injection von einem kräftigen, eventuell mit Schüttelfrost einhergehenden Temperaturanstieg begleitet wird, verhalten sich in dieser Hinsicht die Lungenkranken sehr verschieden. Man kann unter ihnen drei Kategorieen unterscheiden, nämlich solche, die gleich im Beginn oder einige Tage später - bei fortgesetzter Behandlung und steigender Dosis - eine typische Allgemeinreaction darbieten, zweitens solche, bei welchen dieselbe nur mässig ausfällt resp. nur leicht angedeutet ist, und endlich diejenigen, bei denen sie vor der Hand gänzlich fehlt. Von meinen 31 Kranken gehören der ersten Kategorie 9, der zweiten 17, der dritten 5 an. Wovon diese Unterschiede abhängen, lässt sich mit Bestimmtheit nicht sagen, und begnüge $; c h$ mich zunächst mit der Annahme einer individuellen Receptivität gegenüber der allgemein toxischen Wirkung des Mittels, welche ja ihr Analogon in dem Verhalten des menschlichen bezw. thierischen Organismus zu anderen Giftsubstanzen findet. Jedenfalls übt auf den Grad der Allgemeinreaction die In-oder Extensität des Localleidens nicht allein einen ausschlaggebenden Einfluss aus, insofern Kranke mit geringen Lungensymptomen stärker reagiren können, als solche mit ausgebreiteten, andererseits aber natürlich sich auch das Umgekehrte ereignet. Ferner wiederhole ich, dass, wo die Pulsfrequenz wenig Tendenz zum Ansteigen aufweist, in der Regel langsam vermehrte Dosen wenig oder gar keine Temperaturerhöhung verursachen. Ausnahmen hiervon kommen wohl vor, sie sind jedoch nicht häufig.

Ich will Bie nicht davon unterhalten, dass neben den von mir aufgestellten drei Kategorieen Uebergangsfälle vorkommen, bei denen ganz unerwartet und plötzlich erst im späteren Verlaufe der Behandlung einmal eine Temperatursteigerung um 1 oder $2^{0} \mathrm{C}$ sich ereignet, ohne dass eine (gar nicht vorgenommene) Erhöhung der Dosis dafür verantwortlich gemacht werden kann, oder bei denen solche Anstiege sich mehrfach wiederholen. Auch das haben wir gesehen, dass die febrile Reaction sich als Nachwirkung an einem Tage des Pausirens bemerkbar macht, oder dass selbst in ein- und derselben 24stündigen Periode die Temperaturcurve zwei Exacerbationen aufweist. Statt dessen gestatten Sie mir lieber, Ihnen in Form von Curven einige Beispiele der vorher aufgestellten Typen vorzulegen. Besonders bemerkenswerth und als ein Beweis für die von mir geltend gemachte Abhängigkeit der Allgemeinreaction von der individuellen Receptivität erschien mir ein 19jähriges, ziemlich blasses Mädchen mit doppelseitiger Erkrankung der Lungen, bei welchem wir zunächst iiberaus vorsichtig mit der Steigerung der Injectionen vorgingen. $\mathrm{Zu}$ unserem Erstaunen ertrug gerade dieses zarte Kind die Einspritzungen obne jede Zunahme der allabendlich schon vor Beginn der Behandlung sich bis auf $38^{\circ} \mathrm{C}$ erhebenden Temperatur, bis nach Erreichung einer Dosis von 0,01 die Abendtemperatur dauernd um $0,50 \mathrm{C}$ gegenüber der Vorperiode erhöht blieb. Dafür machten sich allerdings des Morgens um so stärkere Remissionen bis auf $36^{0} \mathrm{C}$ und etwas darunter bemerkbar. Das Allgemeinbefinden, sowie der Appetit war zu keiner Zeit alterirt. Eine andere 27 jährige Kranke reagirte während der 16 tägigen bisherigen Behandlungsdauer an fünf Tagen in maximo mit einer Steigeruug der Temperatur von 1 bis $1,5^{0} \mathrm{C}$; die Pulsfrequenz 
erhob sich nie über 96 , so dass schon nach 5 Tagen die Dosis von 0,01 und nach 14 Tagen $0,03 \mathrm{~g}$ erreicht werden konnte. Endlich

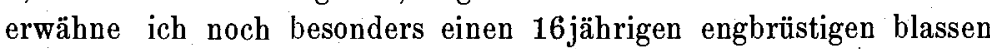
jungen Menschen, welcher, mit dem Residuum einer rechtsseitigen Pleuritis hehaftet, uns zur Beobachtung und probeweisen Behandlung mit dem Koch'schen Mittel in die Anstalt gesandt worden war, obwohl der behandelnde Arzt niemals Auswurf bei ihm beobach tet hatte. Da trotz täglich er Steigerung der Dosis jede fieberhafte Reaction ausblieb, so gelangten wir schon am 7. Tage zu Injectionen von 0,01, wobei sich als interessanter und bedeutungsvoller Effect die Erscheinung einstellte, dass nun Pat. plötzlich geringe Mengen Sputum auszuwerfen begann, welche reichlich Tuberkelbacillen enthielten. Gegenüber diesen Fällen habe ich andere zu verzeichnen, bei denen die Gewöhnung sich so langsam vollzog, dass wir es schliesslich vorzogen, bei jeder einzelnen Dosis, selbst wenn dieselbe keinen bemerkbaren Einfluss auf die Temperaturcurve mehr ausübte, einige Tage zu verharren, bevor wir uns zu erneuter Steigeruug entschlossen.

Je stärker die febrile Reaction, um so intensiver ist die Betheiligung des Allgemeinbefindens. Selbst bei Fehlen der ersteren wird ein gewisser Grad von Abgeschlagenheit nie gänzlich vermisst. Von sonstigen Erscheinungen, die als Ausdruck allgemein toxischer Wirkung aufzufassen sind, wurden folgende beobachtet: Viermal kam es zu leichter, bald vorübergehender Albuminurie, ohne dass die Harnbeschaffenheit eine sonstige Veränderung aufwies. Nur zweimal constatirten wir Vermehrung der Ehrlich'schen Diazoreaction. Bei einem Patienten stellte sich zwei Tage nach dem Aussetzen der Injectionen, welches durch zunehmende Anämie veranlasst wurde (es handelt sich um den Kranken mit hyperplastischen Lymphomen der Cervicaldrüsen), zi em lich intensiver Icterus der Haut und Conjunctiven mit starkem Gallenfarbstoffgehalt des Harns und zeitweiser Entfärbung der Faeces ein. Während auffallender Weise den Arzneiexanthemen zu vergleichende Hautausschläge gar nicht zur Beobachtung kamen, entwickelte sich bei zweien von den zugleich an Laryngophthise leidenden Lungenkranken aphthöse Geschwüre auf der Lippen- und Zungenschleimhaut, welche den Patienten zunächst lebhaften Schmerz verursachten, sich jedoch nach einigen Tagen wieder zurückbildeten. Dass es sich nicht etwa um tuberculöse Geschwüre handelte, wurde, abgesehen von der Beschaffenheit der Geschwüre, durch das Fehlen von Tuberkelbacillen im Secrete derselben bewiesen.

Wiewohl die Behandlungsdauer sich bis zum heutigen Tage bei keinem Kranken über 16 Tage erstreckte, so haben wir doch bei einigen den unzweifelhaften Eindruck einer Besserung des Aussehens und allgemeinen Ernährungszustandes gewonnen, letzteres sich zum Theil auch aussprechend in $\mathrm{Zunahme}$ des Appetites und des Körpergewichts. Bei dem 12jährigen Kinde betrug diese Gewichtsvermehrung 21/2 Pfund, bei zwei anderen Erwachsenen sogar 3 bezw. 5 Pfund, wobei ich noch bemerke, dass diese Kranken sich in relativ günstigen äusseren Verhältnissen befanden, und nicht etwa das Plus lediglich auf Rechnung verbesserter Ernährungsverhältnisse im Krankenhause zu setzen ist. Dagegen vermochten wir bezüglich des Verhaltens der Nachtschweisse keine bestimmten Erfahrungen zu sammeln, da die meisten unserer Kranken, wie mehrfach betont, der Kategorie der „leichten "Fälle angehörten, und Nachtsch weisse nur bei einer Minderzahl von ihnen, zudem in geringem Maasse vorkamen.

Was nun den für uns besonders wichtigen Punkt, die Einwirkung des Koch'schen Mittels auf den Lokalprocess in den Lungen betrifft, so machte sich in der Regel zunächst eine deutliche Vermehrung des Auswurfs bemerkbar, welche bisher nur bei zwei Patienten im weiteren Verlaufe der Behandlung von einer entschiedenen und andauernden Verminderung gefolgt war. Zugleich verband sich mit dieser Zunahme der Quantität des im übrigen unveränderten Sputums ein grösserer Reichthum desselben an Bacillen. Präsentirten sich dieselben vor den Injectionen zum grössten Theil in Form isolirter, über das Gesichtsfeld zerstreuter Exemplare, so waren wir nun oftmals in der Lage, häufchenförmige Anondnung der Bacillen mit dicht gedrängter Aneinanderlagerung derselben wahrzunehmen, wobei die einzelnen Gruppen sich nicht selten aus 10 bis 20 oder noch mehr Stäbchen zusammengesetzt, zeigten. Auch die von Fraentzel betonten Formen der durchbrochenen oder aus perlschnurartig aneinander gereihten Bröckeln bestehenden Formen wurden gesehen; doch sind wir zunächst noch nicht ganz sicher, ob dieselben wirklich als Ausdruck einer Einwirkung des Mittels zu betrachten sind, da ähnliches, wie Fraentzel selbst bemerkt, auch ohne jede Behandlung gar nicht selten beobachtet wird. Wohl aber habe ich bei einigen der von uns untersuchten Sputa den Eindruck gewonnen, als ob die Bacillen sich im ganzen nicht mit jener Intensität tingirten, wie vor den Injectionen. Während sie sonst bei Anwendung von Gabbet's Methode eine lebhaft kirschrothe Farbe annehmen, erschienen sie uns jetzt mehr blassroth und erinnerten in der Beziehung an das Verhalten von Bacterien, welche ihr beginnendes Absterben durch mangelhafte Färbbarkeit verrathen. Indess berichte ich auch über diese Wahrnehmung zunächst mit aller Reserve und ohne die Absicht weiterer Schlussfolgerungen, um so mehr als Koch selbst ja mit hinreichender Schärfe hervorgehoben hat, dass sein Mittel nicht die Tuberkelbacillen als solche, sondern nur das tuberculöse Gewebe abtüdtet. Von einer im späteren Verlaufe statthabenden dauernden Verminderung der Zahl der Bacillen habe ich bei der Kürze der Beobachtungszeit mich bisher nicht mit Sicherheit überzeugen können. Zwar sahen auch wir in vereinzelten Fällen die Bacillen für einen Tag aus dem Sputum schwinden, resp. wir konnten sie in ihm nach Gabbet's Methode nicht mehr nachweisen, aber bereits am nächsten Tage kehrten sie in unverminderter Zahl wieder, und ich zweifle nicht daran, dass die Anwendung des Biedert'schen Verfahrens ihr vorübergehendes Fehlen als ein nur scheinbares dargethan hätte.

Bei einigen Patienten wurden zweifellose Aenderungen der physikalischen Erscheinungen über den Lungen constatirt. So bei einer 31jährigen Frau, welche, an einer mässig ausgebreiteten Verdichtung des linken Oberlappens leidend, die Besonderheit darbot, dass nach Einspritzung des zweiten Milligrammes eine vorher nicht nachweisbare Dämpfung im Bereiche der untersten Rippen der linken Hinterwand mit ziemlich reichlichen daselbst wahrnehmbaren Rasselgeräuschen auftrat. Allem Anscheine nach handelte es sich hier, wie bei den analogen Beobachtungen von Noordens, um eine acute Infiltration bezw. Anschoppung dieses Lungenabschnittes, welche durch die Einspritzungen angefacht wurde und vielleicht als reactiver Vorgang um einen älteren, vordem der physikalischen Untersuchung nicht zugänglichen tuberculösen Herd anzusehen ist. Bei einer zweiten Kranken mit doppelseitiger Affection der Lunge traten im Verlaufe der Behandlung pleuritische Reibegeräusche an einer Stelle des Thorax (vorderer Theil der linken Seitenwand) auf, welche vorher daselbst nicht bestanden hatten. Die Geräusche nahmen regelmässig in den Intervallen zwischen den Injectionen an Intensität $a b$, um jedesmal einige Stunden nach erfolgter neuer Einspritzung, insbesondere in den Abendstunden sich zu verstärken. - Ferner wurde in zwei Fällen mit einseitiger Spitzeninfiltration kurz nach Beginn der Behandlung Rasseln in den oberen Partieen der anderen, nicht ergriffenen Seite wahrgenommen. Eine namhafte Abnahme der acustischen Erscheinungen vermochten wir bisher nur bei drei Patienten festzustellen.

Eine Anzahl der von uns behandelten Patienten endlich bietet nicht blos wegen der Art und Weise, wie sie auf die instituirte Behandlung reagirten, sondern. auch mit Rücksicht auf die Natur und Beschaffenheit der von ihnen dargebotenen Krankheitsprocesse, so bemerkenswerthe Erscheinungen dar, dass ich mir nicht versagen kann, auf ihre Geschichte zum Schluss in etwas ausführlicherer Weise einzugehen :

\section{Doppelseitiger Spitzenkatarrh. Otitis media tuber- culosa dextra.}

26 jähriger, stark abgemagerter Kaufmann. Vor Beginn der Injectionen über beiden Lungenspitzen verhältnissmăssig wenig lauter Schall, unterhalb 
Clavicula dextra deutlichere Dämpfung. Man hört in der rechten Oberund Unterschlüsselbeingrule ziemlich reichliches kleinblasiges Rasseln Desgleichen hinten in Fossa supra- und infraspinata dextra, sowie in Fossa supraspinata sinistra. Seit e'n paar Tagen Stechen und Sausen im rechten Ohr, wozu sich am Tage det Aufnahme, 2. November, Ohrenfluss hinzugesellt hat. Die Untersuchung des Trommelfells ergiebt eine ziemlich grosse Perforation im vorderen unteren Quadranten. Es besteht intermittirendes Fieber mit durchschnittlicher Morgentemperatur von 37,0 und abendlichen Steigerungen bis auf $38,5-39,0^{\circ} \mathrm{C} .^{\circ}$ Die Behandlung begann am 22. Novèmber mit Injection von 0,001 ; da trotz steigender Dosis $39,0^{\circ} \mathrm{C}$ mit Ausnahme eines Tages $\left(39,4^{0} \mathrm{C}\right)$ niemals des abends überschritten wurde, und auch morgens die Temperatur sich dauernd auf $37,0^{\circ} \mathrm{C}$ erhielt, so war bereits am 6. Tage die Dosis 0,01 , und am 13. diejenige von 0,025 erreicht.

Die Eiterung aus dem rechten $\mathrm{Ohr}$ war anfangs eine überaus geringfügige, und der Tuberkelbacillengehalt ein so minimaler, dass in einem ganzen Deckglaspräparate kaum mehr als 1 bis 3 Bacillen aufgefunden werden konnten. Schon nach der ersten Injection erreichte der Ohrenfluss eine während des vorausgegangenen dreiwöchentlichen Krankenhausaufenthaltes nie beobachtete Intensität, und es traten nunmehr massenhaft Bacillen in häufchenförmiger Anordnung in dem eitrigen Secret auf, welche bezüglich ihrer Form und Färbbarkeit keine Abweichung von dem sonstigen Verhalten der Bacillen aufwiesen. Zugleich machte sich vorübergehend ein Gefühl gesteigerter Schmerzhaftigkeit und Schwere in dem erkrankten Ohre bemerkbar, „als wenn ein Fremdkörper darin sässe ${ }^{\star}$. Nach wenigen Tagen nahm indess unter dem Einfluss fortgesetzter Injectionen die Reichlichkeit der Secretion in merkbarer Weise ab, um in den beiden letzten Tagen gänzlich zu versiegen, während der vermehrte Bacillengehalt des Ohreiters bis zuletzt anhielt. Zugleich hat sich die Perforationsöffnung des Trommelfells in augenfälliger Weise verkleinert, an ihren Rändern bemerkt man jetzt frische rothe Granulationen, und die subjectiven Beschwerden seitens des erkrankten Ohres haben gänzlich aufgehört. Eine wesentliche Beeinträchtigung des Hörvermögens hat zu keiner Zeit bestanden. Es liefert daher dieser Fall eine eclatante Bestätigung der Thatsache, dass unter dem Einfluss der Koch'schen Behandlungsmethode ein reichlicher Zerfall tuberculösen Gewebes in Verbindung mit kräftiger entzündlicher Reaction Platz greift.

II. Peritonitis tuberculosa diffusa suppurativa. Nach Koenig erfolgreich operirt. Später Injectionen mit Koch's Mittel.

25jähriger Drechsler, aus hereditår belasteter Familie stammend. Am 25. September zum ersten Male in die Anstalt aufgenommen; 14 Tage vorher plötzlich mit Leibschmerzen, Fieber in Form abendlichen Frierens mit nachfolgendem Schweissausbruch erkrankt. Bei der Aufnahme starke Abmagerung und Erblassung, Lungen frei bis auf beiderseitige Dāmpfung in den hinteren unteren Partieen von der neunten Rippe ab, innerhalb welcher indess nur abgeschwăchtes Vesiculärathmen zu hören ist. Der maximale Umfang des gleichmässig aufgetriebenen und bei Druck, sowie bei der Percussion sehr empfindlichen Abdomens betrăgt anfangs $82 \mathrm{~cm}$, steigt bis zum 10. October bis auf $91 \mathrm{~cm}$. Dabei besteht intermittirendes Fieber mit Morgentemperaturen von $37,0-37,9$ und Abendtemperaturen von 38,0 bis $38,8^{\circ} \mathrm{C}$. Am 10. October Punction des Abdomens, durch welche 3 Liter einer gelblicheitrigen Flüssigkeit entleert werden, welche neben rothen Blutkörperchen und Fettkörnchenzellen sehr viele mononucleäre, einige polynucleäre Leukocyten enthält. Ausserdem (nach vorherigem Sedimentiren und unter Anwendung des Biedert'schen Verfahrens) ziemlich zahlreiche Tuberkelbacillen nachgewiesen. Da das Exsudat schnell von neuem anwāchst, und das Fieber persistirt, wird Patient am 13. October auf die chirurgische Abtheilung verlegt, wo von Herrn Director Ko erte in der Medianlinie ein ausgiebiger Schnitt gemacht und das gesammte Exsudat entleert wird. Nach sorgfältigem Austupfen der Bauchhöhle mit Jodoformschwāmmen wird die Wunde durch Silberdrăhte, welche Peritoneum, Muskulatur und Haut umfassen, geschlossen, und die Haut noch durch eine fortlaufende Seidennath zusammengenäht. Schon am folgenden Tage Abfall des Fiebers, welches von nun ab dauernd sich auf der Norm hält. Schnelle Restitution der Kräfte und Heilung der Wunde durch prima intentio Am 7. November kehrt Patient, welcher bereits seit einigen Tagen wieder umhergeht, auf die innere Abtheilung zurück. Der Umfang des Abdomens be- trügt $75 \mathrm{~cm} . \quad$ Es besteht nur noch eine măssige Empfindlichkeit der linken Regio hypogastrica. Daselbst ist auch der Schall māssig gedạmpft, währẹnd an allen andern Punkten normal lauter Percussionsschall besteht. Am 11. November verlässt Patient das Krankenhaus, tritt aber am 24. November abermals in dasselbe ein, um sich der Behandlung mit dem Kochschen Mittel zu unterwerfen. Es wurde mit einer Injection von 0,004 begonnen, in wenigen Tagen bereits die Dosis 0,05 erreicht. Bis auf eine zeitweise sich bemerkbar machende stärkere Empfindlichkeit des Unterleibes keine Reaction, ebenso bis auf eine einmalige Abendtemperatur von $38,0^{\circ} \mathrm{C}$ kein Fieber!

Dieser Fall ist in zweifacher Beziehung von hervorragendem Interesse, insofern er einen neuen Beitrag zu den bisher vorliegenden Erfahrungen über die Erfolge der chirurgischen Behandlung der tuberculösen Bauchfellentzündung liefert und die Beobachtung bestä. tigt, dass nicht bloss circumscripte tuberculőse Peritonitiden, : sondern auch diffuse Exsudate sich ganz vorzüglich für dieselbe eignen; sodann, weil trotz ziemlich hoher Dosirung der Koch'schen Injectionen eine eigentliche Allgemeinreaction nicht erfolgte, und auch die localen Reizsymptome danach minimale blieben. Die letztere Thatsache würde wohl geeignet sein, uns zu der Annahme zu bewegen, dass hier die Operation den Process nicht bloss zum Stillstand, sondern zu veritabler Ausheilung gebracht habe, wenn nicht die im Vorstehenden mitgetheilten Erfahrungen über das Vorkommen mangelnder Reaction bei anderen tuberculösen Kranken mit noch floriden Affectionen einen solchen Schluss als verfrüht erscheinen liessen. Immerhin darf ich nicht unbetont lassen, dass bei keinem einzigen meiner sämmtlichen übrigen Patienten so hohe Dosen bisher zur Anwendung kamen, wie bei diesem Kranken, und dass es sehr die Frage ist, ob nicht diejenigen von ihnen, welche bisher keine Reaction darboten, bei gleich starken Injectionen doch eine solche - wenigstens vorübergehend aufzuweisen haben würden. ${ }^{1}$ ) Der Werth der operativen Behảndlung, bezüglich deren Wirkungsweise wir nach König's eigenem Ausspruche, , mich in dem vorliegenden Falle um so greifbarer gewesen, als $\mathrm{Pa}$ tient sich kurze Zeit vor dem chirurgischen Eingriff in einem äusserst desolaten Zustande befand, und ohne die Operation allem Anscheine nach binnen kurzem der Ausgang ein ungünstiger gewesen wäre. Ob wir es in Anbetracht nicht nachweisbarer physikalischer Veränderungen an den Lungen hier mit einem jener seltenen Fälle von primärer tuberculöser Peritonitis zu thun hatten, mag füglich dahin gestellt bleiben, da die Frage über das Vorkommen einer solchen vor der Hand überhaupt noch eine strittige ist.

\section{Pseudoleukämie.}

16 jăhriger Schlosserlehrling, am 4. November aufgenommen, litt als Kind an scrophulōsen Drüseneiterungen und bemerkte, dass vor einem Jahre zuerst die rechte, dann die linke Seite seines Halses allmăhlich zu schwellen begann. Zugleich allgemeines Schwächegefühl und Appetitlosigkeit, deren Zunahme schliesslich ihn zur Aufnahme in's Krankenhaus nöthigten. Hierselbst wird ausser hochgradiger Blässe und leicht gelblicher. Verfärbung der Conjunctiven, eine ausserordentliche Anschwellung der beiderseitigen Halsgegend durch Packete von Lymphdrüsen, welche frei beweglich unter der Haut liegen und noch die Abgrenzung der einzelnen, bis zu Borsdorfer Apfel-Grösse herangewachsenen Drüsen gestatten, constatirt. Maximaler Halsumfang $=45 \mathrm{~cm}$. Ausserdem nur noch die rechtsseitigen Achseldrüsen bis zu Haselnussgrösse, und die linksseitigen Leistendrüsen nicht ganz so erheblich geschwollen. Keine Schling- oder Athmungsbeschwerden. Lungen völlig normal, mit Ausnahme einer Abschwächung des Percussionsschalles im Bereiche der 3 letzten rechten unteren Rippen (kleines Pleuraexsudat). Milz palpirbar, Leber mässig vergrössert. Spărliches schleimiges Sputum, in welchem in der Folge keine Tuberkelbacillen gefunden werden. Die Untersuchung gefärbter Blutpräparate (Eosin-Hämatoxylin) ergiebt keine Leukocytenvermehrung. Die vorhandenen weissen Körperchen gehören zum grössten Theil den polynucleären, zum geringeren den kleinen Lymphocyten an. Blutkörperchenzählung nach Z e is s-Th o m a am 12. November $=3050000$, Hāmoglobingehalt nach Fleischl $=20$. Andauerndes, zum Theil remittirendes, zum Theil intermittirendes $\cdot$ Fieber mit Morgentemperaturen von

1) Diese Annahme hat sich inzwischen durch weitere Erfahrungen.als zutreffend erwiesen, während wir andererseits bei dem obigen Kranken. bis zu Einspritzungen von 0,1 ohne wahrnehmbare Reaction gestiegen sind. 
37-37,9 und Abendtemperaturen von 39-40 $\mathrm{C}$. Behandlung besteht zunăchst in innerlicher Darreichung von Pillen auৎ Acid. arsenicos. (bis 0,01 pro die) und tăglichen intraparenchymatösen Doppelinjectionen von 0,005 in die jederseitigen Halsdrüsen. Bis zum 24. November Gesammtarsenverbrauch 0,24175 , unter welcher Beliandlung der Halsumfang bis auf $40 \mathrm{~cm}$ abnimmt. Vom 24. November ab Injectionen nach $\mathrm{Koch}$, beginnend mit 0,001 und an den vier folgenden Tagen steigend auf $0,003,0,005,0,007,0,01$.

Wiewohl es von uns als zweifelhaft angesehen werden musste, $o b$ es sich bei unserem Kranken um Tuberculose als Ursache der Lymphdrüsenhyperplasie handelte, entschlossen wir uns் doch, mit Rücksicht auf đie neuere Mittheilung Waetzoldt's, dass in der That "die chronische Miliartuberculose: der Lymphdrüsen klinische Erscheinungen bedingen kann; welche die grösste Aehnlichkeit mit pseudoleukämischer (Hodgkin'scher) Erkrankung darbietell, zur Instituirung des 'Koch"schen' Verfahrens bei unserem Kranken. Trotż der aùsserordentlichén 'Blässe und körperlichen Schwäche, welche derselbe darbot, wurden die ersten Injectionen mit auffallend gẻringfügiger Allgemein reaction ertragen, nur dass die Abendtemperátur sich um einen halben Grad mehr erhob, und auch die Morgentemperàtur weniger remittirte. Náchđem : aber am 5. Tage, dem 29. November, die Injectionen die Dosis 0,01 erreicht hatten, und wir nunmehr das Mittel zunächst aussetzten, in der Absicht, die zuletzt angewandte Gabe in 2tägigen Intervallen zu wiederholen, trat am 30. November ein so intensiver Icterus in Verbindung mit Albuminurie auf, dass wir auf eime Fortsetzung der Einspritzungen überhaupt verzichten mussten. $O b$ dieser Icterus denjenigen toxischen Formen der Gelbsucht an die Seite zu stellen ist, welche mit vermehrter Zerstörung rother Blutkörperchen und Bildung einer abnorm farbstoffreichen zähflüssigen Galle aus dem in Freiheit gesetzten Blutfarbstoff (Icterus durch Pleinchromie nach Stadelmann) einhergehen, wage ich zunächst nicht zu entscheiden. Nicht gerade zu Gunsten eines solchen Zusammenhanges scheint mir der Umstand zu sprechen, dass der Gehalt des Blutes an rothen Körperchen vom 12. November bis zum 30. November die relativ geringfügige weitere Abnahme von 3050000 bis auf 2793750 erfuhr. Es würde dies eher darauf hinweisen, dass wir es mit einer direkten Einwirkung des Mittels auf die secernirenden Leberzellen zu thun haben, deren Reizung und Schwellung zur Erklärung des Icterus im übrigen völlig hinreichen würde. Seit dem Bestehen der Gelbsucht hat die Färbung der Fäces an Intensität in "solchem Maasse abgenommen, dass sie zeitweise thonfarben erscheinen. Der Umfang des Halses hat sich seit dem 24. November nicht weiter verkleinert. ${ }^{1}$ )

\section{Fünf Fälle von Pleúritis exsudativa sero-fibrinosa.}

Von diesen Fällen betrifft der.eine einen 39jăhrigen sehr kräftigen muskulösen und gut genährten Arbeiter, welcher am 23. August dadurch, dass ihm ein Wagenrad über den Leib ging, ein Trauma der linken Lendengegend davontrug. Die Beschwerden äusserten sich in Schmerzen der linken Nierengegend und wurden von den behandelnden Aerzten des. Krankenhauses Bethanien, in das sich Patient zunächst aufnehmen liess, auf eine Nierenquetschung bezogen. Da trotz vierwöchentlichen Spitalaufenthaltes und darauf

1) Nicht ohne Grund habe ich der Mittheilung dieses Falles die Krankheitsbezeichnung Pseudoleukämie voraufgesetzt, da wăhrend des Druckes meines Vortrages der Patient unter den Erscheinungen zunehmender Schwäche verstorben ist, und die Section ausser den hyperplastischen Lymphomen der Halsdrüsen ebensolche, und zwar sehr umfängliche Vergrösserung der vorderen und hinteren Mediastinal-, der retroperitonealen und mesenterialen, sowie endlich der Beckenlymphdrüsen ergab. Auf dem Durchschnitt erschienen die Drüsen theils von markiger Beschaffenheit und im Zustande beginnender Verfettung, namentlich ihrer Rindensubstanz, theils (besonders an der linken Seite des Halses, woselbst sich auch zu Lebzeiten des Pat. die durch die Arseninjectionen bedingte Verkleinerung besonders bemerkbar machte) von dunkelrother, stellenweise sogar hämorrhagischer Beschaffenheit. .. Ausserdem enthielt die Milz eine grössere Zahl bis bohnengrosser weisslich gefärbter Lymphomknoten. Lungen völlig frei, von Tuberculose nirgend eine Spur, Leberacini, sowie das ganze Organ vergrössert, trübe geschwollen; Ductus choledochus durchẳngig. Gallenblase prall von dunkelgrüner Galle erfültt. folgender 8tägiger Schonzeit der Kranke sich nicht gebessert fühlte, suchte. er am 6. October das städtische Krankenhaus atn Urban auf, woselbst eine linksseitige Pleuritis sero-fibrinosa (Beginn der Dämpfung von der Mitte der Scapula) constatirt wurde. Eine Probepunction erwies das Exsudat als völlig klar, von bräunlicher Färbung, nicht bluthaltig. Patient bot während der ganzen Dauer seines Krankenhausaufenthaltes vom 6 . October bis zum 18. October nicht die leiseste Fieberbewegung dar und fühlte sich bis auf ein Druckgefühl in der linken Seite völlig munter, bei gutem Appetit, und ging umher. Um so interessanter war es uns, dass gerade dieser Kranke, bei welchem wir eine, zunächst nur einfache traumatische Pleuritis diagnosticirt hatten, auf die Einspritzungen des Koch'schen Mittels mit Temperatursteigerungen von über $2 \mathrm{Grad}$ und ziemlich ausgesprochener Beeinträchtigung des Allgemeinbefindens reagirte. Wir begannen die Behandlung mit einer Injection von 0,005 und stiegen allerdings schon am vierten Tage mit Rücksicht auf den vortrefflichen Ernährungszustand bis auf 0,02. In den Dämpfungsverhältnissen hat sich bisher keine Veränderung vollzogen, trotzdem Patient sich jetzt am 16. Behandlungstage befindet und allmählich auf die Dosis 0,035 gekommen ist. Es erhebt sich hier die natürlich schwer zu lösende Frage, ob die Pleuritis in Anbetracht der ausgesprochenen Allgemeinreaction. bei allerdings fehlender Veränderung der Localsymptorne (keine erheblichere Schmerzzunahme, resp. Vergrösserúng oder Verkleinerung der Dämpfung) als eine tuberculöse anzusehen ist oder nicht. Träfe das erstere zu, so würde man das Trauma in unserem Falle als ein Gelegenheitsmoment, welches die Ansiedlung der Tuberkelbacillen in der gequetschten Pleura begünstigte, anzusehen haben und den Fall seiner Entwicklung nach in Parallele mit den Beobachtungen M. Mendelsohn's u. a. über das Vorkommen, bezw. die Entwickelung tuberculöser Lungenphthise auf der Basis von Traumen zu setzen haben. - Ein zweiter unserer mit Pleuritis behafteten Patienten leidet zugleich an Lungentuberculose und bietet nichts Bemerkenswerthes dar; ein dritter, ohne das manifeste Bestehen dieser Complication, hat bisher zu wenig auf die Einspritzungen reagirt, um Schlüsse auf die Grundursache seines Leidens zu ziehen. Dagegen constatirten wir bei den beiden restirenden vou den fünf Kranken, einem 16jährigen Bureaudiener, dessen ich schon oben einmal gedachte, und einem 30jährigen Buchbinder das Auftreten von Tuberkelbacillen im Sputum erst, nachdem die Behandlungsmethode nach Koch begonnen, und die Injectionen eine Anzahl von Tagen fortgesetzt waren. Der eine der beiden Patienten hatte bis zu seiner Aufnahme in's Krankenhaus überhaupt nicht expectorirt und begann erst auszuwerfen, nachdem die Dosenhöhe von 0,01 erreicht war; in dem Sputum des zweiten, welcher an Pleuritis duplex leidet, konnten wir selbst nach Biedert's Methode vorher keine Bacillen auffinden, bis am vierten Tage der Injectionen $(0,004)$ dieselben ziemlich zahlreich auftraten. Zugleich wurde als Effect der weiteren Behandlung eine deutliche Verkleinerung des doppelseitigen Exsudates wahrgenommen, ohne dass Patient besonders lebhafte Allgemeinreaction aufwies. Es bedarf keines weiteren Hinweises, in welchem Maasse Beobachtungen, wie diese, der Auffassung das Wort reden, dass ein grosser Theil der früher auf unbekannte Ursachen oder lediglich auf Refrigeration hiusichtlich ihrer Entstehung bezogenen serös-fibrinösen Pleuraexsudate tuberculöser Natur sind.

\section{Fall von Diabetes mit tuberculöser Lungenphthise.}

Patient, ein 52jăhriger Rentier, war uns von dem ihn behandeluden Arzte

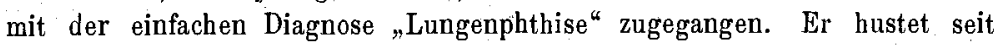
3 Jahren, weiss nicht, dass er zugleich an Diabetes leidet, und bietet die Erscheinungen einer nicht allzu beschränkten doppelseitigen Spitzeninfiltration. Trotz ziemlicher Magerkeit ist das Allgemeinbefinden ein günstiges; es bestand in den letzten Monaten nie Fieber. Gelegentlich der Harnuntersuchung wurde zufällig von uns der Zuckergehalt des Harns entdeckt, welcher $1 \%$ betrug.

In Anbetracht der doppelseitigen Lungenaffection und des complicirenden Diabetes, sowie der Angabe des Kranken, dass er zeitweise an Herzklopfen gelitten habe, glaubten wir hier, wo allem 
Anschein nach das Lungenleiden sich auf der Basis des Diabetes entwickelt hat, mit den Injectionen besonders vorsichtig zu Werke gehen zu müssen, und begannen mit Einspritzung eines halben Milligramms. Aber selbst, seitdem wir wegen der completen Reactionslosigkeit täglich die Dosis um ein ganzes Milligramm verstärkt und auf diese Weise jctzt bereits 0,01 erreicht haben, hat sich noch keine Spur von Temperaturerhöhung oder Aenderung des günstigen Allgemeinbefindeus, der Pulsfrequenz u. s. w. bemerkbar gemacht, ein neuer Beweis der wiederholt betonten Abhängigkeit der Allgemeinerscheinungen von der individuellen Empfänglichleit der Patienten. 